\title{
Performance of Compact Cotton Genotypes under High Density Planting System in Irrigated Ecosystem
}

\author{
Mohan Chavan*, Satyanarayana Rao, B. K. Desai and B. G. Koppalkar \\ Department of Agronomy, Agricultural College, UAS, Raichur-584104 \\ (Karnataka State), India \\ *Corresponding author
}

\section{Keywords \\ Cotton genotypes, Density planting system, Irrigated ecosystem \\ Article Info \\ Accepted: \\ 05 January 2020 \\ Available Online: \\ 10 February 2020}

\section{A B S T R A C T}

A field experiment entitled "Performance of compact cotton genotypes under high density planting system in irrigated condition" was conducted at Agricultural College, Raichur during Kharif 2016-17 and 2017-18 on medium black soil, neutral in nature with low available nitrogen, medium phosphorus and high in potassium. The experiment was laid out in split plot design with three compact cotton genotypes viz., $\mathrm{G}_{1}$ : SCS-1206, $\mathrm{G}_{2}$ : DSC-99 and $\mathrm{G}_{3}$ : Suraj as main plot treatments and three planting geometries viz., $S_{1}: 60 \mathrm{~cm} \mathrm{x} 10 \mathrm{~cm}, S_{2}: 75 \mathrm{~cm} \times 10$ $\mathrm{cm}$ and $\mathrm{S}_{3}: 90 \mathrm{~cm} \times 10 \mathrm{~cm}$ as sub plot treatments and it was compared with conventional system of cotton cultivation with Bt cotton hybrid ATM with recommended spacing of $90 \mathrm{~cm} \times 60 \mathrm{~cm}$. Among the different compact cotton genotypes, $\mathrm{G}_{1}$ : SCS-1206 recorded highest seed cotton yield (2886 $\mathrm{kg} \mathrm{ha}^{-1}$ ) followed by genotype $\mathrm{G}_{3}$ : Suraj $\left(2754 \mathrm{~kg} \mathrm{ha}^{-1}\right)$ which were significantly superior over genotype $\mathrm{G}_{2}$ : DSC-99 $\left(2486 \mathrm{~kg}^{-1}\right)$. Among the different planting geometries, a closer row spacing of $S_{1}: 60 \mathrm{~cm} \times 10 \mathrm{~cm}$ recorded significantly higher seed cotton yield (2896 kg ha-1) over a medium row spacing of $S_{2}: 75 \mathrm{~cm} \mathrm{x} 10 \mathrm{~cm}$ $\left(2758 \mathrm{~kg} \mathrm{ha}^{-1}\right)$ and significantly lower seed cotton yield was recorded with a wider row spacing of $S_{3}: 90 \mathrm{~cm} \times 10 \mathrm{~cm}\left(2472 \mathrm{~kg} \mathrm{ha}^{-1}\right)$. Among the different combinations, the genotype $\mathrm{G}_{1}$ : SCS-1206 grown at $S_{1}: 60 \mathrm{~cm} \times 10 \mathrm{~cm}$ spacing recorded significantly higher seed cotton yield $\left(3096 \mathrm{~kg} \mathrm{ha}^{-1}\right)$ and it was found at par with the combination of same genotype $\mathrm{G}_{1}: S C S-1206$ with $\mathrm{S}_{2}: 75 \mathrm{~cm}$ x $10 \mathrm{~cm}$ spacing $\left(2949 \mathrm{~kg} \mathrm{ha}^{-1}\right)$ and genotype $\mathrm{G}_{3}$ : Suraj with $\mathrm{S}_{1}: 60 \mathrm{~cm}$ x $10 \mathrm{~cm}$ spacing $\left(2923 \mathrm{~kg} \mathrm{ha}^{-1}\right)$. cotton grown under conventional system with Bt cotton hybrid ATM at a recommended spacing of $90 \mathrm{~cm}$ x $60 \mathrm{~cm}$ recorded significantly lower seed cotton yield $\left(2314 \mathrm{~kg} \mathrm{ha}^{-1}\right)$ when compared with the all the treatment combinations of cotton grown under HDPS except with the combinations of genotype $\mathrm{G}_{2}$ : DSC-99 at $\mathrm{S}_{2}: 75 \mathrm{~cm} \times 10 \mathrm{~cm}$ spacing and genotype $\mathrm{G}_{2}$ : DSC-99 at $\mathrm{S}_{3}$ : $90 \mathrm{~cm}$ x $10 \mathrm{~cm}$ spacing (2525 and $2263 \mathrm{~kg} \mathrm{ha}^{-1}$, respectively). 


\section{Introduction}

The concept HDPS is widely adopted by several countries such as China, Brazil, Uzbekistan, Australia, Argentina and several other countries where in plant population of $1,00,000$ to $2,00,000$ per hectare is maintained and high seed cotton yield of 40 to 90 quintals per hectare is realized. HDPS is more relevant to India to establish sustainable production system as the productivity of cotton is low in India. Compact cotton type of genotypes have the advantage of short sympodial branches with reduced inter-nodal length giving morphological feature of compressed habit and clustered boll habit on account of low vertical and horizontal growth it occupies minimum space. The HDPS cotton not only provides scope for double cropping and mechanized harvesting but also has the added advantage of requiring few pickings only. Therefore, which in turn reduces the labour cost as well as seed cost as farmers will use the varietal seeds during next sowing season.

Therefore, the present investigation was undertaken to find out the suitable compact cotton genotypes and planting geometry with a view to achieve high yield levels under irrigated ecosystem of North Eastern Dry zone of Karnataka.

\section{Materials and Methods}

A field experiment entitled "Performance of compact cotton genotypes under high density planting in irrigated ecosystem" was conducted at Agricultural College, UAS, Raichur during Kharif 2016-17 and 2017-18 on medium black soil, neutral in nature with low available nitrogen, medium phosphorus, rich in potassium. The climatic condition during experimental period was favorable and regular irrigation was provided to crop during both the years at later part of crop growth stages i.e, from 60 DAS to till first picking.
The experiment was laid out in split plot design with three compact cotton genotypes viz., $\mathrm{G}_{1}$ : SCS-1206, G 2 : DSC-99, G 3 : Suraj as main plot treatments and three planting geometries viz., $\mathrm{S}_{1}: 60 \mathrm{~cm}$ x $10 \mathrm{~cm}(1,66,666$ plants $\left.\mathrm{ha}^{-1}\right), \mathrm{S}_{2}: 75 \mathrm{~cm}$ x $10 \mathrm{~cm}(1,33,333$ plants $\left.\mathrm{ha}^{-1}\right)$ and $\mathrm{S}_{3}: 90 \mathrm{~cm} \times 10 \mathrm{~cm}(1,11,111$ plants $\left.\mathrm{ha}^{-1}\right)$ as sub plot treatments along with conventional system of cotton cultivation with Bt cotton hybrid ATM at a recommended spacing of $90 \mathrm{~cm} \times 10 \mathrm{~cm}$ (uneven control)

\section{Results and Discussion}

\section{Genotypes}

Among the different compact cotton genotypes, $\mathrm{G}_{1}$ : SCS-1206 recorded higher seed cotton yield $\left(2886 \mathrm{~kg} \mathrm{ha}^{-1}\right.$ on pooled basis) followed by genotype $\mathrm{G}_{3}$ : Suraj (2754 $\mathrm{kg} \mathrm{ha}^{-1}$ ) and which were significantly higher when compared with genotype $\mathrm{G}_{2}$ : DSC-99 (2486 $\mathrm{kg} \mathrm{ha}^{-1}$ ). This difference in seed cotton yield was mainly attributed to significant difference in yield components viz., number of bolls per plant (12.82 and 12.12, respectively on pooled basis), boll weight (3.73 and $3.54 \mathrm{~g}$, respectively on pooled basis) and seed cotton yield per plant (25.98 and $24.40 \mathrm{~g}$, respectively on pooled basis) and which was further due difference in growth attributes. Similar results were also reported by Udikeri and Shashidhara (2017), Ajaykumar et al. (2017) and Sankaryanana et al. (2018).

\section{Planting geometry}

Difference in seed cotton yield due to different planting geometry was evident. Among different row spacings, a closer spacing of $\mathrm{S}_{1}: 60 \mathrm{~cm} \times 10 \mathrm{~cm}$ recorded significantly higher seed cotton yield (2896 $\mathrm{kg} \mathrm{ha}^{-1}$ on pooled basis) when compared with the medium row spacing of $S_{2}: 75 \mathrm{~cm} \times 10 \mathrm{~cm}$ (2758 $\mathrm{kg} \mathrm{ha}^{-1}$ on pooled basis) and wider row 
spacing of $\mathrm{S}_{3}: 90 \mathrm{~cm} \mathrm{x} 10 \mathrm{~cm}\left(2472 \mathrm{~kg} \mathrm{ha}^{-1}\right.$ on pooled basis). This differences in seed cotton yield was attributed to higher plant population per unit area even though the growth and yield attributes were lower when compared to recorded under medium and wider row spacings and increase in the number of plants per unit area could be compensated for decrease in yield components per plant under narrow spacing. Significantly superior seed cotton yield observed was mainly due to higher number of harvested bolls and higher plants population per unit area as supported findings of Alur (2016) and Devi et al. (2018).

Table.1 Plant growth attributing characters of compact cotton genotypes under high density planting system

\begin{tabular}{|c|c|c|c|c|c|c|c|c|c|}
\hline \multirow[t]{2}{*}{ Treatments } & \multicolumn{3}{|c|}{ Plant height (cm) } & \multicolumn{3}{|c|}{ Sympodials/plant } & \multicolumn{3}{|c|}{$\begin{array}{l}\text { Total dry matter production } \\
\qquad\left(\text { g plant }^{-1}\right)\end{array}$} \\
\hline & 2016-17 & 2017-18 & Pooled & 2016-17 & 2017-18 & Pooled & 2016-17 & 2017-18 & Pooled \\
\hline \multicolumn{10}{|c|}{ Main plots (G) } \\
\hline $\mathbf{G}_{1}$ & $128.96^{\mathrm{a}}$ & $114.12^{\mathrm{a}}$ & $121.54^{\mathrm{a}}$ & $13.00^{\mathrm{a}}$ & $12.44^{\mathrm{a}}$ & $12.72^{\mathrm{a}}$ & $130.52^{\mathrm{a}}$ & $122.92^{\mathrm{a}}$ & $126.72^{a}$ \\
\hline $\mathbf{G}_{2}$ & $121.89^{b}$ & $95.96^{\mathrm{b}}$ & $108.92^{b}$ & $11.29^{b}$ & $10.91^{b}$ & $11.10^{\mathrm{b}}$ & $112.65^{c}$ & $103.89^{b}$ & $108.27^{\mathrm{c}}$ \\
\hline $\mathbf{G}_{3}$ & $133.31^{\mathrm{c}}$ & $120.51^{\mathrm{a}}$ & $126.91^{\mathrm{a}}$ & $12.47^{\mathrm{a}}$ & $11.98^{\mathrm{a}}$ & $12.22^{\mathrm{a}}$ & $125.69^{b}$ & $116.88^{\mathrm{a}}$ & $121.29^{b}$ \\
\hline S.Em \pm & 2.28 & 1.78 & 1.77 & 0.25 & 0.23 & 0.19 & 1.18 & 1.82 & 1.20 \\
\hline \multicolumn{10}{|l|}{ Sub plots (S) } \\
\hline $\mathbf{S}_{1}$ & $137.09^{\mathrm{a}}$ & $120.58^{\mathrm{a}}$ & $128.83^{\mathrm{a}}$ & $9.91^{\mathrm{c}}$ & $9.56^{\mathrm{c}}$ & $9.73^{c}$ & $110.86^{c}$ & $101.26^{\mathrm{c}}$ & $106.06^{c}$ \\
\hline $\mathbf{S}_{\mathbf{2}}$ & $129.67^{b}$ & $111.84^{b}$ & $120.75^{b}$ & $11.98^{b}$ & $11.64^{b}$ & $11.81^{b}$ & $121.41^{b}$ & $112.93^{b}$ & $117.17^{b}$ \\
\hline $\mathbf{S}_{\mathbf{3}}$ & $117.40^{\mathrm{a}}$ & $98.18^{c}$ & $107.79^{c}$ & $14.87^{\mathrm{a}}$ & $14.13^{\mathrm{a}}$ & $14.50^{\mathrm{a}}$ & $136.60^{\mathrm{a}}$ & $129.49^{\mathrm{a}}$ & $133.05^{\mathrm{a}}$ \\
\hline S.Em \pm & 1.78 & 2.34 & 2.31 & 0.36 & 0.26 & 0.28 & 1.52 & 1.63 & 1.08 \\
\hline \multicolumn{10}{|c|}{ Interactions $(\mathbf{G} \times \mathbf{S})$} \\
\hline $\mathbf{G}_{1} \mathbf{S}_{1}$ & $139.13^{\mathrm{a}}$ & $125.46^{\mathrm{ab}}$ & $132.30^{\mathrm{ab}}$ & $10.33^{\mathrm{de}}$ & $10.13^{\text {ef }}$ & $10.23^{\mathrm{ef}}$ & $118.08^{\mathrm{cd}}$ & $108.95^{\mathrm{cd}}$ & $113.52^{d}$ \\
\hline $\mathbf{G}_{1} \mathbf{S}_{2}$ & $129.40^{a-c}$ & $114.39^{b c}$ & $121.90^{b-}$ & $12.93^{\mathrm{c}}$ & $12.20^{\mathrm{cd}}$ & $12.57^{\mathrm{c}}$ & $129.38^{b}$ & $121.64^{b}$ & $125.51^{b}$ \\
\hline $\mathbf{G}_{1} \mathbf{S}_{3}$ & $118.33^{\mathrm{cd}}$ & $102.52^{\mathrm{cd}}$ & $110.43^{\mathrm{de}}$ & $15.73^{\mathrm{a}}$ & $15.00^{\mathrm{a}}$ & $15.37^{\mathrm{a}}$ & $144.10^{\mathrm{a}}$ & $138.16^{\mathrm{a}}$ & $141.13^{\mathrm{a}}$ \\
\hline $\mathbf{G}_{2} \mathbf{S}_{1}$ & $130.80^{\mathrm{a}-\mathrm{c}}$ & $106.47^{\mathrm{cd}}$ & $118.63^{\mathrm{cd}}$ & $9.47^{\mathrm{e}}$ & $8.80^{f}$ & $9.13^{\mathrm{f}}$ & $102.30^{\mathrm{e}}$ & $91.39^{\mathrm{e}}$ & $96.85^{\mathrm{f}}$ \\
\hline $\mathbf{G}_{2} \mathbf{S}_{2}$ & $123.40^{\mathrm{b}-\mathrm{d}}$ & $97.54^{d}$ & $110.47^{\mathrm{de}}$ & $10.73^{\text {de }}$ & $10.87^{\mathrm{de}}$ & $10.80^{\mathrm{de}}$ & $110.46^{d}$ & $102.72^{d}$ & $106.59^{e}$ \\
\hline $\mathbf{G}_{2} \mathbf{S}_{3}$ & $111.47^{\mathrm{d}}$ & $83.86^{\mathrm{e}}$ & $97.66^{\mathrm{e}}$ & $13.67^{\mathrm{bc}}$ & $13.07^{\mathrm{bc}}$ & $13.37^{\mathrm{bc}}$ & $125.20^{b c}$ & $117.57^{b c}$ & $121.39^{b c}$ \\
\hline $\mathbf{G}_{3} \mathbf{S}_{1}$ & $141.33^{\mathrm{a}}$ & $129.8^{\mathrm{a}}$ & $135.57^{\mathrm{a}}$ & $9.93^{\mathrm{e}}$ & $9.73^{\mathrm{et}}$ & $9.83^{\text {et }}$ & $112.21^{d}$ & $103.45^{d}$ & $107.83^{\text {de }}$ \\
\hline $\mathbf{G}_{3} \mathbf{S}_{2}$ & $136.20^{\mathrm{ab}}$ & $123.57^{\mathrm{ab}}$ & $129.89^{\mathrm{a}-\mathrm{c}}$ & $12.27^{\mathrm{cd}}$ & $11.87^{\mathrm{cd}}$ & $12.07^{\mathrm{cd}}$ & $124.38^{b c}$ & $114.44^{\mathrm{bc}}$ & $119.41^{c}$ \\
\hline $\mathbf{G}_{3} \mathbf{S}_{3}$ & $122.40^{\mathrm{cd}}$ & $108.16^{\mathrm{cd}}$ & $115.28^{\mathrm{d}}$ & $15.20^{\mathrm{ab}}$ & $14.33^{\mathrm{ab}}$ & $14.77^{\mathrm{ab}}$ & $140.49^{\mathrm{a}}$ & $132.75^{\mathrm{a}}$ & $136.62^{a}$ \\
\hline S.Em \pm & 3.95 & 4.05 & 3.99 & 0.63 & 0.45 & 0.49 & 2.64 & 2.83 & 1.87 \\
\hline Control & 119.07 & 106.30 & 112.69 & 20.73 & 19.27 & 20.00 & 180.00 & 171.06 & 175.53 \\
\hline S.Em \pm & 3.64 & 3.72 & 3.67 & 0.57 & 0.44 & 0.45 & 2.70 & 2.89 & 1.83 \\
\hline $\mathrm{CD}(\mathrm{P}=\mathbf{0 . 0 5})$ & 10.82 & 11.05 & 10.92 & 1.69 & 1.30 & 1.34 & 8.01 & 8.59 & 5.43 \\
\hline
\end{tabular}


Table.2 Yield attributing characters of compact cotton genotypes under high density planting system

\begin{tabular}{|c|c|c|c|c|c|c|c|c|c|}
\hline \multirow[t]{2}{*}{ Treatments } & \multicolumn{3}{|c|}{ Number of bolls/plant } & \multicolumn{3}{|c|}{ Boll weight (g) } & \multicolumn{3}{|c|}{$\begin{array}{l}\text { Seed cotton yield/plant } \\
\text { (g) }\end{array}$} \\
\hline & 2016-17 & 2017-18 & Pooled & 2016-17 & $\begin{array}{c}2017- \\
18\end{array}$ & Pooled & 2016-17 & 2017-18 & Pooled \\
\hline \multicolumn{10}{|c|}{ Main plots (G) } \\
\hline $\mathbf{G}_{1}$ & $13.22^{\mathrm{a}}$ & $12.42^{\mathrm{a}}$ & $12.82^{\mathrm{a}}$ & $3.80^{\mathrm{a}}$ & $3.66^{\mathrm{a}}$ & $3.73^{\mathrm{a}}$ & $26.42^{\mathrm{a}}$ & $25.55^{\mathrm{a}}$ & $25.98^{\mathrm{a}}$ \\
\hline $\mathbf{G}_{2}$ & $11.04^{\mathrm{b}}$ & $10.20^{\mathrm{b}}$ & $10.62^{\mathrm{b}}$ & $3.35^{\mathrm{b}}$ & $3.29^{b}$ & $3.32^{\mathrm{c}}$ & $22.90^{c}$ & $21.32^{\mathrm{c}}$ & $22.11^{\mathrm{c}}$ \\
\hline $\mathbf{G}_{3}$ & $12.56^{\mathrm{a}}$ & $11.69^{\mathrm{a}}$ & $12.12^{\mathrm{a}}$ & $3.64^{\mathrm{a}}$ & $3.54^{\mathrm{a}}$ & $3.59^{\mathrm{b}}$ & $24.97^{\mathrm{b}}$ & $23.82^{\mathrm{b}}$ & $24.40^{\mathrm{b}}$ \\
\hline S.Em \pm & 032 & 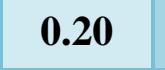 & 024 & 005 & 005 & 03 & 027 & 032 & 0.16 \\
\hline \multicolumn{10}{|l|}{ Sub plots (S) } \\
\hline $\mathbf{S}_{1}$ & $10.42^{c}$ & $9.40^{\mathrm{c}}$ & 9.91 & $3.36^{\mathrm{c}}$ & $3.25^{\mathrm{b}}$ & $3.30^{\mathrm{b}}$ & $23.18^{c}$ & $21.61^{\mathrm{c}}$ & $22.40^{\mathrm{c}}$ \\
\hline $\mathbf{S}_{2}$ & $12.07^{\mathrm{b}}$ & $11.29^{b}$ & $11.68^{b}$ & $3.49^{\mathrm{b}}$ & $3.44^{\mathrm{b}}$ & $3.46^{\mathrm{b}}$ & $24.47^{\mathrm{b}}$ & $23.39^{\mathrm{b}}$ & $23.93^{\mathrm{b}}$ \\
\hline $\mathbf{S}_{3}$ & $14.33^{\mathrm{a}}$ & $13.62^{\mathrm{a}}$ & $13.98^{\mathrm{a}}$ & $3.95^{\mathrm{a}}$ & $3.80^{\mathrm{a}}$ & $3.88^{\mathrm{a}}$ & $26.64^{\mathrm{a}}$ & $25.68^{\mathrm{a}}$ & $26.16^{\mathrm{a}}$ \\
\hline S.Em \pm & 0.21 & & 19 & 0.03 & 0.11 & 006 & 0.35 & 0.46 & 0.26 \\
\hline \multicolumn{10}{|c|}{ Interactions $(\mathbf{G} \times \mathbf{S})$} \\
\hline $\mathbf{G}_{1} \mathbf{S}_{1}$ & $11.13^{\mathrm{c}}$ & $10.13^{\mathrm{c}}$ & $10.63^{c}$ & $3.58^{\mathrm{bc}}$ & $3.41^{\mathrm{a}-\mathrm{c}}$ & $3.50^{\mathrm{bc}}$ & $25.06^{b-d}$ & $23.63^{\mathrm{bc}}$ & $24.35^{\mathrm{cd}}$ \\
\hline $\mathbf{G}_{1} \mathbf{S}_{2}$ & $13.07^{\mathrm{b}}$ & $12.33^{\mathrm{b}}$ & $12.70^{\mathrm{b}}$ & $3.71^{\mathrm{bc}}$ & $3.69^{\mathrm{ab}}$ & $3.70^{\mathrm{ab}}$ & $25.94^{\mathrm{bc}}$ & $25.40^{\mathrm{ab}}$ & $25.67^{\mathrm{bc}}$ \\
\hline $\mathbf{G}_{1} \mathbf{S}_{3}$ & $15.47^{\mathrm{a}}$ & $14.80^{\mathrm{a}}$ & $15.13^{\mathrm{a}}$ & $4.12^{\mathrm{a}}$ & $3.89^{\mathrm{a}}$ & $4.00^{\mathrm{a}}$ & $28.26^{\mathrm{a}}$ & $27.6^{\mathrm{a}}$ & $27.93^{\mathrm{a}}$ \\
\hline $\mathbf{G}_{2} \mathbf{S}_{1}$ & $9.40^{\mathrm{d}}$ & $8.47^{\mathrm{d}}$ & $8.93^{\mathrm{d}}$ & $3.06^{\mathrm{e}}$ & $3.01^{\mathrm{c}}$ & $3.03^{\mathrm{d}}$ & $21.15^{\mathrm{f}}$ & $19.37^{\mathrm{d}}$ & $20.26^{\mathrm{f}}$ \\
\hline $\mathbf{G}_{2} \mathbf{S}_{2}$ & $10.73^{c}$ & $9.93^{c}$ & $10.33^{c}$ & $3.27^{\mathrm{de}}$ & $3.19^{\mathrm{bc}}$ & $3.23^{\mathrm{cd}}$ & $22.77^{\mathrm{ef}}$ & $21.13^{\text {cd }}$ & $21.95^{\mathrm{e}}$ \\
\hline $\mathbf{G}_{2} \mathbf{S}_{3}$ & $13.00^{\mathrm{b}}$ & $12.20^{\mathrm{b}}$ & $12.60^{\mathrm{b}}$ & $3.73^{b}$ & $3.66^{\mathrm{ab}}$ & $3.70^{\mathrm{ab}}$ & $24.77^{\mathrm{cd}}$ & $23.44^{\mathrm{bc}}$ & $24.11^{\mathrm{d}}$ \\
\hline $\mathbf{G}_{3} \mathbf{S}_{1}$ & $10.73^{c}$ & $9.60^{c}$ & $10.17^{c}$ & $3.44^{\mathrm{cd}}$ & $3.32^{\mathrm{a}-\mathrm{c}}$ & $3.38^{\mathrm{bc}}$ & $23.34^{\mathrm{de}}$ & $21.82^{\mathrm{cd}}$ & $22.58^{\mathrm{e}}$ \\
\hline $\mathbf{G}_{3} \mathbf{S}_{2}$ & $12.40^{\mathrm{b}}$ & $11.60^{\mathrm{b}}$ & $12.00^{\mathrm{b}}$ & $3.47^{\mathrm{b}-\mathrm{d}}$ & $3.43^{\mathrm{a}-\mathrm{c}}$ & $3.45^{\mathrm{bc}}$ & $24.68^{c-e}$ & $23.64^{\mathrm{bc}}$ & $24.16^{\mathrm{d}}$ \\
\hline $\mathbf{G}_{3} \mathbf{S}_{3}$ & $14.53^{\mathrm{a}}$ & $\begin{array}{c}13.8 \\
7^{\mathrm{a}}\end{array}$ & $14.20^{\mathrm{a}}$ & $4.02^{\mathrm{a}}$ & $3.86^{\mathrm{a}}$ & $3.94^{\mathrm{a}}$ & $26.89^{\mathrm{ab}}$ & $26.00^{\mathrm{ab}}$ & $26.45^{\mathrm{b}}$ \\
\hline & & 0.36 & & 0.00 & & & 0.00 & 0.79 & 0.45 \\
\hline Control & 37.07 & 35.99 & 36.53 & 4.32 & 4.24 & 4.28 & 143.45 & 138.14 & 140.80 \\
\hline S.Em \pm & 0.50 & 0.41 & 0.43 & 0.09 & 0.17 & 0.08 & 1.37 & 0.88 & 0.77 \\
\hline $\mathrm{CD}(\mathrm{P}=\mathbf{0 . 0 5})$ & 1.47 & 1.21 & 1.26 & 0.28 & 0.50 & 0.24 & 4.08 & 2.49 & 2.29 \\
\hline
\end{tabular}


Table.3 Yield and economics of compact cotton genotypes under high density planting system

\begin{tabular}{|c|c|c|c|c|c|c|c|c|c|}
\hline \multirow[t]{2}{*}{ Treatments } & \multicolumn{3}{|c|}{$\begin{array}{l}\text { Seed cotton yield (kg } \\
\left.\text { ha }^{-1}\right)\end{array}$} & \multicolumn{3}{|c|}{ Net returns ( $\square$ ha $^{-1}$ ) } & \multicolumn{3}{|c|}{ B C Ratio } \\
\hline & $\begin{array}{l}2016- \\
17\end{array}$ & $\begin{array}{l}2017- \\
18\end{array}$ & Pooled & 2016-17 & 2017-18 & Pooled & 2016-17 & 2017-18 & Pooled \\
\hline \multicolumn{10}{|c|}{ Main plots (G) } \\
\hline $\mathbf{G}_{1}$ & $2962^{\mathrm{a}}$ & $2811^{\mathrm{a}}$ & $2886^{\mathrm{a}}$ & $84064^{\mathrm{a}}$ & $78629^{a}$ & $81346^{\mathrm{a}}$ & $2.81^{\mathrm{a}}$ & $2.64^{\mathrm{a}}$ & $2.73^{\mathrm{a}}$ \\
\hline $\mathbf{G}_{2}$ & $2584^{\mathrm{b}}$ & $2388^{\mathrm{b}}$ & $2486^{\mathrm{b}}$ & $67407^{b}$ & $59609^{b}$ & $63508^{\mathrm{b}}$ & $2.45^{\mathrm{b}}$ & $2.24^{\mathrm{b}}$ & $2.35^{\mathrm{b}}$ \\
\hline $\mathbf{G}_{3}$ & $2842^{\mathrm{a}}$ & $2666^{\mathrm{a}}$ & $2754^{\mathrm{a}}$ & $78764^{\mathrm{a}}$ & $72104^{a}$ & $75434^{a}$ & $2.70^{\mathrm{a}}$ & $2.51^{\mathrm{a}}$ & $2.60^{\mathrm{a}}$ \\
\hline S.Em & .58 & $\mathbf{5 2 . 7 7}$ & 53.73 & 2445 & 2375 & 2390 & 0.06 & 0.05 & 0.05 \\
\hline \multicolumn{10}{|l|}{ Sub plots (S) } \\
\hline $\mathbf{S}_{1}$ & $2974^{\mathrm{a}}$ & $2819^{\mathrm{a}}$ & $2896^{\mathrm{a}}$ & $83661^{a}$ & $78068^{\mathrm{a}}$ & $80865^{\mathrm{a}}$ & $2.77^{\mathrm{a}}$ & $2.60^{\mathrm{a}}$ & $2.69^{\mathrm{a}}$ \\
\hline $\mathbf{S}_{2}$ & $2841^{\mathrm{b}}$ & $2675^{\mathrm{b}}$ & $2758^{\mathrm{b}}$ & $78840^{\mathrm{a}}$ & $72624^{\mathrm{a}}$ & $75732^{\mathrm{a}}$ & $2.71^{\mathrm{a}}$ & $2.52^{\mathrm{a}}$ & $2.61^{\mathrm{a}}$ \\
\hline $\mathbf{S}_{\mathbf{3}}$ & $2573^{c}$ & $2371^{c}$ & $2472^{c}$ & $67734^{\mathrm{b}}$ & $59650^{\mathrm{b}}$ & $63692^{b}$ & $2.49^{\mathrm{b}}$ & $2.27^{\mathrm{b}}$ & $2.38^{\mathrm{b}}$ \\
\hline S.Em \pm & 42.38 & 43.68 & 42.87 & 1865 & 1966 & 190 & 0.03 & 0.05 & 0.03 \\
\hline \multicolumn{10}{|c|}{ Interactions $(\mathbf{G} \times \mathbf{S})$} \\
\hline $\mathbf{G}_{1} \mathbf{S}_{\mathbf{1}}$ & $3156^{\mathrm{a}}$ & $3035^{\mathrm{a}}$ & $3096^{\mathrm{a}}$ & $91669^{\mathrm{a}}$ & $87803^{\mathrm{a}}$ & $89736^{a}$ & $2.94^{\mathrm{a}}$ & $2.80^{\mathrm{a}}$ & $2.87^{\mathrm{a}}$ \\
\hline $\mathbf{G}_{\mathbf{1}} \mathbf{S}_{2}$ & $3020^{\mathrm{ab}}$ & $2879^{\mathrm{ab}}$ & $2949^{\mathrm{ab}}$ & $86706^{\mathrm{ab}}$ & $81819^{a b}$ & $84263^{\mathrm{ab}}$ & $2.88^{\mathrm{a}}$ & $2.71^{\mathrm{a}}$ & $2.80^{\mathrm{ab}}$ \\
\hline $\mathbf{G}_{\mathbf{1}} \mathbf{S}_{3}$ & $2711^{\mathrm{cd}}$ & $2518^{\mathrm{cd}}$ & $2615^{\text {cd }}$ & $73816^{\mathrm{cd}}$ & $66265^{\mathrm{cd}}$ & $70041^{\mathrm{cd}}$ & $\underset{d}{2.62^{b-}}$ & $2.41^{\mathrm{bc}}$ & $2.52^{\mathrm{cd}}$ \\
\hline $\mathbf{G}_{2} \mathbf{S}_{1}$ & $2761^{\text {cd }}$ & $2579^{\text {cd }}$ & $2670^{\text {cd }}$ & $74304^{\text {cd }}$ & $67283^{\text {cd }}$ & $70793^{\text {cd }}$ & $2.57^{\mathrm{cd}}$ & $2.38^{\mathrm{bc}}$ & $2.48^{\mathrm{cd}}$ \\
\hline $\mathbf{G}_{\mathbf{2}} \mathbf{S}_{\mathbf{2}}$ & $2621^{\mathrm{d}}$ & $2428^{d}$ & $2525^{\mathrm{d}}$ & $69180^{d}$ & $61524^{d}$ & $65352^{d}$ & $2.50^{\mathrm{d}}$ & $2.29^{\text {cd }}$ & $2.39^{\mathrm{d}}$ \\
\hline $\mathbf{G}_{\mathbf{2}} \mathbf{S}_{\mathbf{3}}$ & $2368^{\mathrm{e}}$ & $2157^{\mathrm{e}}$ & $2263^{\mathrm{e}}$ & $58739^{\mathrm{e}}$ & $50020^{\mathrm{e}}$ & $54379^{\mathrm{e}}$ & $2.29^{\mathrm{e}}$ & $2.06^{\mathrm{d}}$ & $2.18^{\mathrm{e}}$ \\
\hline $\mathbf{G}_{3} \mathbf{S}_{1}$ & $3005^{\mathrm{ab}}$ & $2842^{\mathrm{ab}}$ & $2923^{\mathrm{ab}}$ & $85010^{\mathrm{ab}}$ & 791 & $82064^{\mathrm{ab}}$ & $2.80^{\mathrm{ab}}$ & $2.62^{\mathrm{ab}}$ & $2.71^{\mathrm{ab}}$ \\
\hline $\mathbf{G}_{3} \mathbf{S}_{2}$ & $2882^{\mathrm{bc}}$ & $2717^{\mathrm{bc}}$ & $2799^{\mathrm{bc}}$ & $80634^{\mathrm{bc}}$ & $74529^{\mathrm{bc}}$ & $77582^{\mathrm{bc}}$ & $\underset{c}{2.75^{\mathrm{a}-}}$ & $\underset{c}{2.56^{\mathrm{a}-}}$ & $2.65^{\mathrm{bc}}$ \\
\hline $\mathbf{G}_{3} \mathbf{S}_{3}$ & $2639^{\text {cd }}$ & $2438^{\mathrm{d}}$ & $2539^{d}$ & $70648^{\text {cd }}$ & $62665^{\mathrm{d}}$ & $66657^{\text {cd }}$ & $2.55^{\mathrm{d}}$ & $2.33^{\mathrm{c}}$ & $2.44^{\mathrm{d}}$ \\
\hline S.Em \pm & 73.40 & 75.65 & 74.25 & 3230 & 3404 & 33.05 & 0.07 & 0.08 & 0.06 \\
\hline Control & 2419 & 2208 & 2314 & 57848 & 49195 & 53522 & 2.19 & 1.98 & 2.09 \\
\hline S.Em \pm & 77 & 78 & 75 & 3383 & 3488 & 3331 & 0.07 & 0.07 & 0.07 \\
\hline $\mathrm{CD}(\mathrm{P}=\mathbf{0 . 0 5})$ & 228 & 230 & 222 & 10052 & 10364 & 9898 & 0.22 & 0.22 & 0.21 \\
\hline
\end{tabular}




\section{Interaction effect}

Interaction effect of compact cotton genotypes and planting geometries were found significant. Among the different combinations, interaction of genotype $\mathrm{G}_{1}$ : SCS -1206 with a row spacing of $S_{1}: 60 \mathrm{~cm} \mathrm{x}$ $10 \mathrm{~cm}$ recorded significantly higher seed cotton yield (3096 kg ha ${ }^{-1}$ on pooled basis) when compared to rest of treatment combination. However, it remained at par with the combination of $\mathrm{G}_{1}$ : SCS-1206 with a row spacing of $S_{2}: 75 \mathrm{~cm} \mathrm{x} 10 \mathrm{~cm}(2949 \mathrm{~kg}$ $\mathrm{ha}^{-1}$ on pooled basis) and genotype $\mathrm{G}_{3}$ : Suraj with a row spacing of $S_{1}: 60 \mathrm{~cm} \times 10 \mathrm{~cm}$ (2923 kg ha ${ }^{-1}$ on pooled basis). Further, cotton grown under conventional system with $\mathrm{Bt}$ cotton hybrid ATM at a recommended spacing of $90 \mathrm{~cm} \quad$ x $60 \mathrm{~cm}$ recorded significantly lower seed cotton yield $(2314 \mathrm{~kg}$ $\mathrm{ha}^{-1}$ on pooled basis) when compared with all the treatment combinations of cotton grown under HDPS except with the combination of genotype $\mathrm{G}_{2}$ : DSC-99 with a row spacing of $\mathrm{S}_{2}: 75 \mathrm{~cm} \mathrm{x} 10 \mathrm{~cm}\left(2525 \mathrm{~kg} \mathrm{ha}^{-1}\right.$ on pooled basis) and genotype $\mathrm{G}_{2}$ : DSC-99 with a row spacing of $\mathrm{S}_{3}: 90 \mathrm{~cm} \times 10 \mathrm{~cm}\left(2263 \mathrm{~kg} \mathrm{ha}^{-1}\right.$, on pooled basis). The results are in line with the findings of Tuppad (2015) and Parlawar et al. (2017).

\section{Economics}

Among the different compact cotton types, genotype $\mathrm{G}_{1}$ : SCS-1206 and $\mathrm{G}_{3}$ : Suraj recorded significantly higher net returns ( $\square$ 81,346 and 75,434 ha $^{-1}$ respectively on pooled basis) and $\mathrm{BC}$ ratio (2.73 and 2.60, respectively on pooled basis). While the genotype $\mathrm{G}_{2}$ : DSC-99 recorded significantly lower net returns $\left(\square 63,508 \mathrm{ha}^{-1}\right.$ on pooled basis) and $\mathrm{BC}$ ratio (2.35 on pooled bases). Among different planting geometries, a closer row spacing of $S_{1}: 60 \mathrm{~cm} \times 10 \mathrm{~cm}$ recorded significantly higher net returns $\left(\square 80,865 \mathrm{ha}^{-1}\right.$ on pooled basis) and $\mathrm{BC}$ ratio (2.69 on pooled basis) and it was found at par with medium row spacing of $S_{2}: 75 \mathrm{~cm} \mathrm{x} 10 \mathrm{~cm}(\square 75,732$ $\mathrm{ha}^{-1}$ and 2.61, respectively on pooled basis). While, wider row spacing of $S_{3}: 90 \mathrm{~cm} \times 10$ $\mathrm{cm}$ recorded significantly lower net returns and BC ratio ( $\square$ 63,692 $\mathrm{ha}^{-1}$ and 2.38, respectively on pooled basis). Among different interactions of cotton grown under HDPS, a combination of genotype $\mathrm{G}_{1}$ : SCS1206 with row spacing of $S_{1}: 60 \mathrm{~cm} \times 10 \mathrm{~cm}$ recorded significantly higher net returns and BC ratio ( $\square$ 89,736 $\mathrm{ha}^{-1}$ and 2.87, respectively on pooled basis) and found on par with combination of genotype $\mathrm{G}_{1}$ : SCS1206 with a spacing of $S_{2}: 75 \mathrm{~cm} \mathrm{x} 10 \mathrm{~cm}$ ( $\square$ $84,263 \mathrm{ha}^{-1}$ and 2.80 , respectively on pooled basis) and genotype $S_{3}$ : Suraj with a row spacing of $S_{1}: 60 \mathrm{~cm} \times 10 \mathrm{~cm}\left(\square 82,064 \mathrm{ha}^{-1}\right.$ and 2.71, respectively on pooled basis). Significantly lower net returns and BC ratio ( $\square 54,379 \mathrm{ha}^{-1}$ and 2.18 , respectively on pooled basis) was observed with combination of genotype $\mathrm{G}_{2}$ : DSC-99 with a row spacing of $\mathrm{S}_{3}: 90 \mathrm{~cm} \times 10 \mathrm{~cm}$. Cotton grown under conventional system with $\mathrm{Bt}$ cotton hybrid ATM with a recommended spacing of $90 \mathrm{~cm}$ x $60 \mathrm{~cm}$ recorded significantly lower economic values ( $\square 53,522 \mathrm{ha}^{-1}$ and 2.09, respectively on pooled basis) when compared with the cotton grown under HDPS. This result was supported by findings of Tuppad (2015) and UdiKeri (2017).

\section{References}

Udikeri, M. and Shashidhar, G. B., 2017, Influence of different planting geometry and fertilizer levels on yield and economics of compact cotton genotypes. Inter. J. Agril. Sci., 9(33): 4482-4485.

Ajayakumar, M. Y., Umesh, M. R., Shivalila and Nidagundi, J. M., 2017, Light interception and yield response of cotton varieties to high density planting and fertilizers in sub-tropical India. $J$. 
Appl. \& Nat. Sci., 9(3): 1835-1839.

Sankarnarayanan, K., Singh, J. and Rajendran, K., 2018, Identification of suitable high density planting system genotypes its response to different levels of fertilizers compared with Bt cotton. J. Cotton Res. Dev., 32(1): 8496.

Alur A, 2016, Studies on high density planting and nutrient management in compact cotton genotypes. M. Sc (Agri) Thesis, Univ. Agric. Sci., Raichur (India).

Devi, B., Bharathi, S., Rekha, S. M. and Jayalalitha, K., 2018, Nutrient uptake and economics of cotton in high density planting system under varied plant spacing and nitrogen levels. J. Res. ANGRAU, 46(1): 26-29.

Parlawar, N. D., Jiotode, D. J., Khawle, V. S., Kubde, K. J. and Puri, P. D., 2017, Effect of planting geometry and varieties on morpho-physiological parameters and yield of cotton. J. Soils and Crops., 27(2):152-158.

Tuppad, G. B., 2015, Response of compact cotton genotypes to graded levels of fertilizers under varied planting density and defoliators. Ph. D. (Agri) Thesis, Univ. Agric. Sci., Dharwad (India).

\section{How to cite this article:}

Mohan Chavan, Satyanarayana Rao, B. K. Desai and Koppalkar, B. G. 2020. Performance of Compact Cotton Genotypes under High Density Planting System in Irrigated Ecosystem. Int.J.Curr.Microbiol.App.Sci. 9(02): 166-172. doi: https://doi.org/10.20546/ijcmas.2020.902.020 